\title{
Sampling philosophy of medicine
}

Philippe Huneman, Gérard Lambert and Marc Silberstein (eds.) Classification, Disease and Evidence: New Essays in the Philosophy of Medicine. Dordrecht, Heidelberg, New York, London: Springer, 2015, 232pp, \$129 HB

\section{Brendan Clarke \\ b.clarke@ucl.ac.uk}

Department of Science and Technology Studies University College London Gower Street London WC1E 6BT UK

This volume is a collection of philosophy of medicine chapters that deal, in one way or another, with the three linked themes of the title. The approach is predominantly analytic, and thus the book fits into the tradition that Caplan (1992) calls philosophy of medicine (rather than biomedical ethics or medical humanities). However, the primarily analytic aspect of the chapters does not exclude related ways of working, with several of the papers touching on ethical themes. The chapters are well researched and written, and provide the reader with a useful snapshot of contemporary philosophy of medicine.

The first part of the introduction consists of a useful contextualisation of the emergence of this field (vii-x). The editors characterise philosophy of medicine as "deeply shaped by three independent sources" (viii), viz.: conceptual analysis; second, advances in biology that permitted the identification of mechanisms of disease; third, the development of statistical methods for assessing the causes of disease, and the efficacy of treatments directed against them. This part of the introduction is excellent, and well worth reading. However, the second half of the introduction is less easy to praise. My major criticism of this volume concerns the tangled way that the editors have chosen to frame a series of "Questions in the Philosophy of Medicine" (xi), and then linked them to themes discussed in the chapters. For the specialist (very much the intended audience), these brief remarks don't add much. For the generalist, on the other hand, these remarks will be more confusing than clarifying. Happily, however, the volume title alone provides a sufficiently clear way of distinguishing the remits of the chapters, with themes of classification (Singy, Dekeuwer, Méthot and Alizon), disease (Méthot and Alizon again, Cunningham, Lemoine, Murphy, Demazeux) and evidence (Coste, Giroux and Stegenga) being readily apparent. I will deal with them here in that order.

Singy's chapter (Power, Knowledge, and Laughter: Forensic Psychiatry and the Misuse of the DSM) begins with a familiar argument concerning the non-objectivity of the DSM. However, the second part of the chapter is much more interesting. Here, Singy argues, even if the DSM were somehow to become fully objective, we should still be cautious when using it outside the clinic. His argument focuses on the role of the DSM in forensic psychiatry, where (as the title suggests) he believes it to be seriously misused. His argument is that psychiatry's object of study shifts as it moves between the clinic and the courtroom. The DSM is (arguably) suited to the first of these locales, because it deals with diseases. However, the law deals with incapacities, and as incapacities are not diseases, the DSM is unsuited to this second setting. The demands of these different contexts suggest that developing distinct ways of classifying in the clinic and the courtroom would be "beneficial for both parties." (140). The argument is built within a Foucaultian framework, and while Singy suggests that "Psychiatry can make you laugh" (132) the conclusions of this chapter suggest that there's not much to smile about here for those interested in classification in medico-legal practice. 
Dekeuwer's chapter (Defining Genetic Disease) will mainly be of interest to philosophers of biology, rather than medicine. Here, the emphasis is firmly on the genetic, rather than the disease. This chapter investigates why genes are taken as the primary causes of some disease. Given that other causes are involved in the pathogenesis of these conditions (such as environmental factors), why is this the case? Beginning with a thoughtful analysis of Magnus' argument that none of three definitions of genetic disease - direct causal action, covariance of gene and trait, and the possible manipulability of genetic cause - are adequate alone, she concludes that a combination of the three might be.

Méthot and Alizon's chapter is a key-stone in this collection. Schematically, it presents an analysis of the way in which knowledge claims about emerging diseases are made and justified (95-6) using the case of the 1918-1919 influenza pandemic. The authors present "two distinct scientific styles of practice" (96) which may be used to understand the pandemic, which they call the ecologicalevolutionary and the molecular pathology frameworks. Importantly, each gives partial and distinct explanations of disease phenomena. The chapter is heavily detail-driven, and bears favourable comparison to Hacking's work on styles of scientific thinking.

Cunningham's chapter argues for a rejection of the popular distinction between the "art" and "science" of medicine. Cunningham argues that this distinction is harmful - particularly because it confuses the important role of values medicine. However, the grounds for the distinction are not spelled out as clearly as might be liked, and this blunt foil unfortunately robs this chapter of much of its rhetorical force. But it's hard to disagree with the conclusion that it is helpful to regard medicine "as an integrative science aiming at multi-level explanation in the service of patient health" (15).

As the introduction to this collection also suggests, definition of disease has become a canonical problem for philosophers of medicine. Lemoine's chapter discusses issues that might be raised by attempts to naturalise disease concepts. Lemoine argues that philosophers have attempted to define a pre-naturalised concept of disease, and the first half of the chapter is largely devoted to a clear discussion of the causes and consequences of this (24-8). The second half then aims to sketch out what a naturalised definition of disease might look here. The aim is ambitious: medically, by analogy with the theorising of mechanics in the later enlightenment, by pointing out the possible advantages of a general theory of disease. Philosophically, too, this chapter exercises an effective rejoinder to those philosophers of medicine who are satisfied by working with toy examples, rather than engaging more strenuously with practice.

Murphy's chapter tells a story about the illusory possibilities and power of reductionism to inform us about the causal structure of psychiatry. An excessive focus on neuroscience, argues Murphy, "gets in the way of more promising approaches" (43) in psychiatry. More psychology, especially, is needed: there are things that just can't be done via neuroscience alone. For instance, Murphy argues that psychiatric diseases have been crammed into a generic medical model (44ff) that "tells us what our explanatory task is" (56). This seems to be an eliminativist trajectory, where we run the risk that "neuroscience will crowd out the psychology" in psychiatry (58). Murphy argues that this is troubling - why should an epistemology given and driven by neuroscience trump other ways of understanding the mind?

The next chapter argues to a similar conclusion to the preceding pair. Demazeux argues that we should probably "give up the idea of setting a definite biological criterion of the normal and the pathological for all mental disorders" (90). The significance of this claim is developed by reference to Boorse's and Wakefield's differing accounts of disease. Demazeux does well to make the important differences between the two perspicuous to the non-specialist while arguing that each, in their own way, are unified by their invocation of some idea of mental functions. 
Coste's chapter is a contribution to the scanty literature on diagnosis. What, asks Coste, are the pros and cons of different kinds of reasoning in diagnosis? Here, three approaches - Bayesian, causal, and pathophysiological - are presented, and related to an underlying nosology. The argument is that, first, these three different strategies for diagnostic reasoning are under tension and, second, that these different strategies might only be applicable to some kinds of disease. Most of the work of this second argument requires reference to Coste's earlier work, in which his nosology is set out. But the review of styles of reasoning in diagnosis is well worth having.

The continuing use of the term 'risk factor' in epidemiology presents those interested in causality in medicine with something of a conceptual headache. Viewed in terms of the traditional distinction between correlation and causation, risk factors are neither fish nor fowl. For example, some risk factors (like smoking for heart disease) seem causal because they license intervention. On the other hand, other risk factors (like height for breast cancer) seem to be correlations, in that no plausible causal mechanism seems to link the risk factor with the outcome. Giroux's chapter presents an extremely useful review of the concept of the risk factor that includes a short but valuable historical section that traces the development of this concept.

QATs (quality assessment tools) are simple tools used to judge the quality of evidence arising from clinical trials. As Stegenga argues, the use of QATs is rising, and they present advantages over the previously-popular technique of determining quality of evidence as largely arising from the methodology used to produce it. A paradigmatic example of this practice was the use of evidence hierarchies, in which evidence from a randomised control trial would trump that produced by an observational study. The problem here was that this way of ranking evidence worked along a single axis only, and a poorly designed RCT would trump an impeccable observational study. The QATs that Stegenga discusses can rank trials using many different metrics, and thus provide a way of circumventing the difficulties of evidence hierarchies. However, as is usual with novel techniques, they bring new conceptual difficulties. Here, Stegenga highlights the "underdetermination of evidential significance" (205) associated with them: there are very many QATs, each of which has low inter-rater (different operators, same QAT) and inter-tool (same operator, different QATs) reliabilities. This is an essential chapter for those interested in current developments in EvidenceBased Medicine.

By way of conclusion, this volume is an excellent set of chapters, rather than a cohesive review of the subject. Read in this way, this book is a valuable addition to the research literature.

\section{Reference}

Caplan, Arthur. 1992. Does the philosophy of medicine exist? Theoretical Medicine and Bioethics 13(1): 67-77. doi: 10.1007/BF00489220 